\title{
Sparsity-constrained three-dimensional image reconstruction for C-arm angiography
}

\author{
Essam A. Rashed ${ }^{\mathrm{a}, *}$, Mohammad al-Shatouri $^{\mathrm{b}}$, Hiroyuki Kudo ${ }^{\mathrm{c}, \mathrm{d}}$ \\ ${ }^{a}$ Image Science Lab., Department of Mathematics, Faculty of Science, Suez Canal \\ University, Ismailia 41522, Egypt \\ ${ }^{b}$ Department of Radiology, Faculty of Medicine, Suez Canal University, Ismailia 41522, \\ Egypt \\ ${ }^{c}$ Division of Information Engineering, Faculty of Engineering, Information and Systems, \\ University of Tsukuba, Tsukuba 305-8573, Japan \\ ${ }^{d}$ JST, ERATO, Quantum-Beam Phase Imaging Project, Katahira 2-1-1, Aoba-ku, Sendai \\ 980-8577, Japan
}

\begin{abstract}
$\mathrm{X}$-ray $\mathrm{C}$-arm is an important imaging tool in interventional radiology, roadmapping and radiation therapy because it provides accurate descriptions of vascular anatomy and therapeutic end point. In common interventional radiology, the C-arm scanner produces a set of two-dimensional (2D) X-ray projection data obtained with a detector by rotating the scanner gantry around the patient. Unlike conventional fluoroscopic imaging, three-dimensional (3D) C-arm computed tomography (CT) provides more accurate cross-sectional images, which are helpful for therapy planning, guidance and evaluation in interventional radiology. However, 3D vascular imaging using the conventional C-arm fluoroscopy encounters some geometry challenges. Inspired by the theory of compressed sensing, we developed an image reconstruction algorithm for conventional angiography C-arm scanners. The main challenge
\end{abstract}

\footnotetext{
${ }^{*}$ Corresponding author E-mail address: erashed@science.suez.edu.eg (E.A. Rashed).
} 
in this image reconstruction problem is the projection data limitations. We consider a small number of views acquired from a short rotation orbit with offset scan geometry. The proposed method, called sparsity-constrained angiography (SCAN), is developed using the alternating direction method of multipliers, and the results obtained from simulated and real data are encouraging. SCAN algorithm provides a framework to generate 3D vascular images using the conventional C-arm scanners in lower cost than conventional 3D imaging scanners.

Keywords: Image reconstruction, computed tomography, C-arm angiography, sparsity, ADMM

\section{Introduction}

C-arm angiography is an effective imaging tool for many different types of physicians such as cardiologist, radiologist, surgeons, urologist, orthopedic surgeries and neurovascular applications. It is an important tool in interventional surgery, radiation therapy, road mapping and guidance [1]. Imageguided interventional radiology aims to provide physicians with anatomical and physiological structure of the patient organs during minimally invasive surgery procedures. In typical protocols for imaging blood-vessels, the patient is injected with contrast agent, and the acquired two-dimensional (2D) images are processed through digital subtraction angiography (DSA) approach. In DSA, an image acquired before the injection of the contrast agent is subtracted from a contrast-based image to remove surrounding complicated structures [2]. Sample images acquired for kidney tumor vessels using the DSA approach are shown in Fig. 1. The common challenge in this procedure 
is that 2D images are insufficient to provide required three-dimensional (3D) information of vessel anatomy due to complicated structure and the incapability to identify overlapping vessels (Fig. 1(c)). In clinical procedures, such as transarterial chemoemobolization (TACE), physicians usually change the angle to acquire the image by rotating the C-arm gantry to a different view (Fig. 1(d)) and mentally process these images to understand the 3D vessel structure. This procedure is known to increase the patient dose of both the x-ray and the contrast agent acquisitions. Moreover, it requires special clinical experience to be processed successfully. In some applications, the C-arm is placed close to a computed tomography (CT) scanner in order to perform a CT scan simultaneously, which is the common way to observe the 3D structure of vessels using CT images. However, this approach also increases the patient dose and patient is required to be moved from the surgery table to the scanner table which is inconvenient in some cases. Three-dimensional C-arm CT employ 2D x-ray projection data obtained with a detector by rotating the $\mathrm{C}$-arm around the patient to reconstruct CT-like images. The number of required projections is approximately several hundred of views. Modern multi-axis C-arm systems can provide 3D images by recording several projections around the patient and perform tomographic image reconstruction. However, these advanced systems are expensive compared to standard C-arm scanners [1].

In this paper, we present the experience of using conventional 2D C-arm scanners to provide high-quality 3D vascular images. The main module in the proposed framework is an image reconstruction algorithm from limited projection data. We consider data limitations for a challenging geometry 
(a) Image without contrast agent

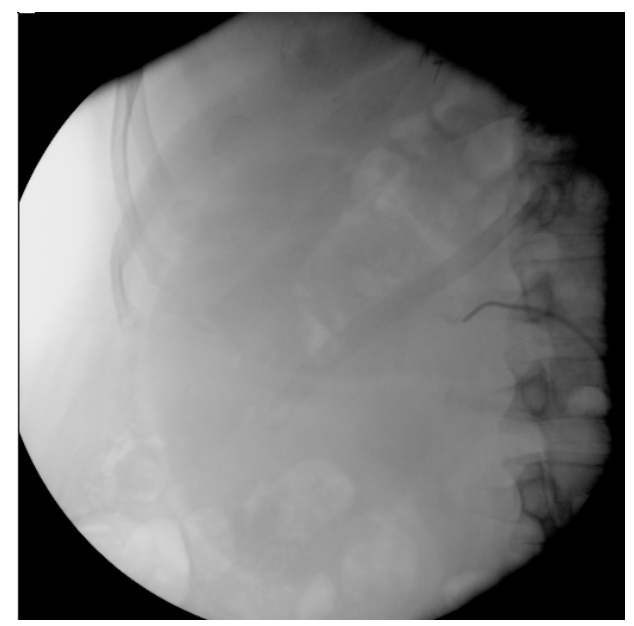

(c) DSA image

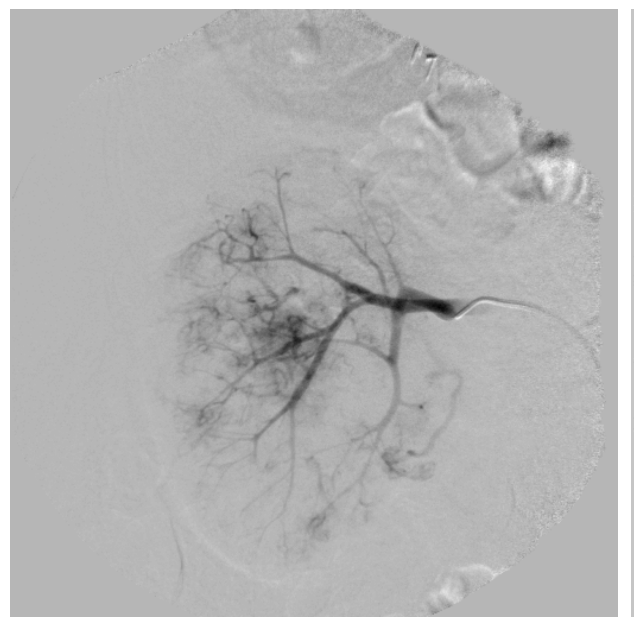

(b) Image with contrast agent

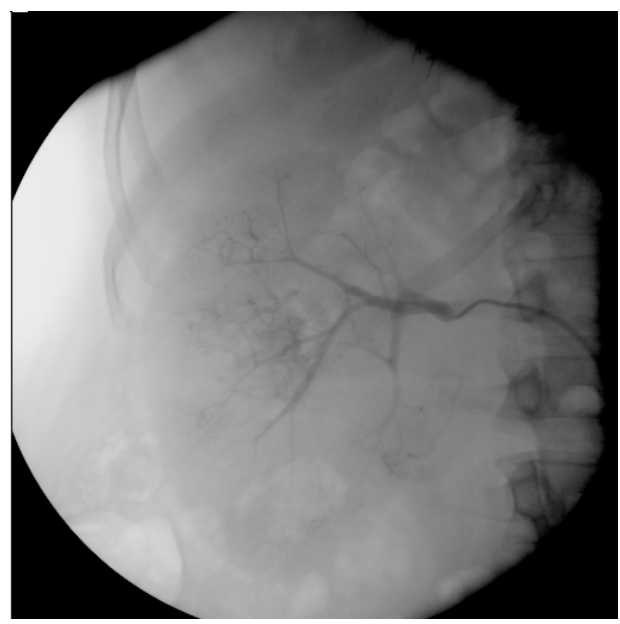

(d) DSA image (another view)

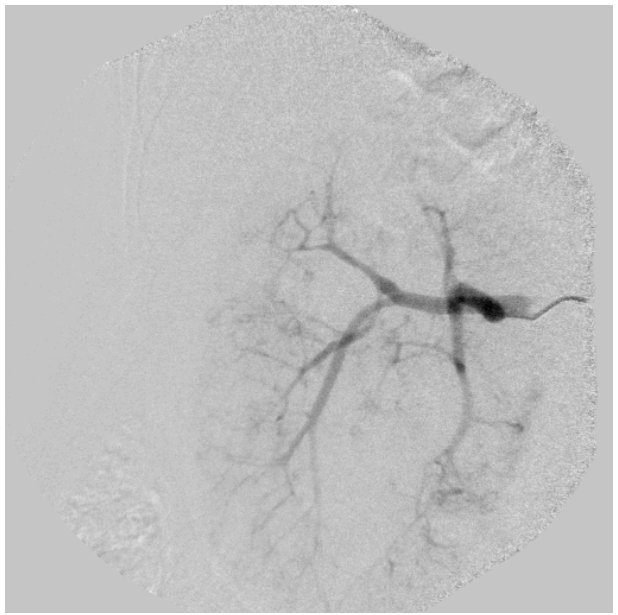

Figure 1: Digital subtraction angiography (DSA) technique used to visualize the arteries of a kidney tumor. (a) and (b) are the images acquired before and after the injection of contrast agent, respectively. (c) is the DSA image obtained by subtracting (a) from (b). (d) is a DSA image obtained from a different view angle. Images are acquired during transarterial embolization session in Suez Canal University Specialized Hospital. 
setup, where the projection data is only available from a small number of projection views measured over a limited rotation orbit with non-isocenteric C-arm scanner. Recent work presented several algorithms to tackle image reconstruction for limitation problems discussed in this paper (e.g. [3-11]). A successful experiment to modify the mobile isocenteric C-arm system to include a flat-panel detector towards cone-beam CT imaging is presented in [6]. Results demonstrate sufficient image quality for guidance of interventional procedures. In another study, a CT data acquisition system using commercial C-arm system was developed [7]. The developed system was calibrated to determine geometrical parameters and FDK algorithm was used for cone-beam image reconstruction. Ritter et al. developed a clinical prototype mobile C-arm for 3D soft tissue imaging [9]. The proposed system facilitates several interventional procedures.

The proposed approach is based on the theory of compressed sensing (CS) $[12,13]$ by introducing a sparsity-constrained cost function for image reconstruction. The image reconstruction algorithm is developed to handle sparse objects such as blood-vessels, where most of image pixel values are zeros or close to zeros and only small number of image pixels have non-zeros intensities. The proposed algorithm, called sparsity-constrained angiography (SCAN), is derived using the alternating direction method of multipliers (ADMM) with emphasis on the sparsity nature of the angiography imaging. ADMM is an optimization method developed in the 1970s [14]. Since then, it has been used in several areas such as image processing, machine learning and distributed optimization. Very recently, ADMM was used to develop a statistical iterative algorithm for $\mathrm{CT}$ image reconstruction $[15,16]$. The pro- 
posed method is evaluated through experimental studies including computer simulation and real-data. Achieved result demonstrates a notable artifacts reduction and image quality improvement compared to conventional image reconstruction techniques such as ART.

The rest of this paper is organized as follows. In Section 2, we introduce the scanner geometry and data acquisition for the system under study. The proposed method is presented in Section 3 together with a brief introduction to related mathematical concepts. Experimental studies are discussed and analyzed in Section 4 and the paper is concluded in Section 5.

\section{Scanner geometry and data acquisition}

In this section, we introduce the C-arm system used in this study together with geometry configurations that limit the 3D image reconstruction and produce challenges arising from data limitations. The device used in this study is a clinical C-arm scanner (Ziehm Vision R, Ziehm Imaging GmbH, Nürnberg, Germany ${ }^{1}$ ), installed in Suez Canal University Specialized Hospital (SCUSH). This scanner is equipped with an image intensifier with diameter of $31 \mathrm{~cm}$. The tube current and potential range from 1.5 to $75 \mathrm{~mA}$ and 40 to $120 \mathrm{kVp}$, respectively. Orbital movement range is about $115^{\circ}$, source to detector distance is $97 \mathrm{~cm}, \mathrm{C}$-arm depth is $68 \mathrm{~cm}$ and magnitude of detector offset is about $13 \mathrm{~cm}$. The C-arm system generates images in $1024 \times 1024$ pixel matrix with pixel size of about $0.03 \times 0.03 \mathrm{~cm}$. Unless noted otherwise, experimental studies presented here employ the system configuration

\footnotetext{
${ }^{1}$ http://www.ziehm.com/en/products/ziehm-vision-r/
} 
parameters of real scanner stated above.

This system can provide 2D radiographic and fluoroscopic images for minimal invasive surgery with manual control of gantry movement. However, 3D imaging using the scanner under study has several data limitation problems. First, the scanner gantry is manually operated, so we can acquire only a small number of projection views (see Fig. 2(b)). Unlike 3D systems, where the gantry motion is automatically controlled, the manual movements are known to be inaccurate and gantry positioning is likely to have some displacement errors. Moreover, the manual gantry movement can produce only a small number of projection views. Measuring only a small number of projections possesses a great benefit to reduce the accumulated patient dose. However, the corresponding image reconstruction problem becomes unstable and achieving exact reconstruction is difficult. Second, the gantry rotation orbit is limited to a range of angles less than those theoretically required for stable CT reconstruction. The imaging system used in this work has a rotation range limited to $115^{\circ}$, which is below the normal angular range used in CT reconstruction (see Fig. 2(c)). Third, the C-arm scanner is nonisocenteric. Accordingly, the x-ray source and detector are shifted from the central line that passes through the gantry isocenter as shown in Fig. 2(d). This setup is common in C-arm scanners to increase the scanner FOV in 2D fluoroscopy. However, it is an uncommon setup for 3D image reconstruction. It is known that, in the offset scan geometry, the common region which can be viewed in all projection data is rather small and thus most of projection data suffer from data truncation. To illustrate the data limitation problems of the imaging geometry under study, we show the imaging configuration of 
(a) Full scan

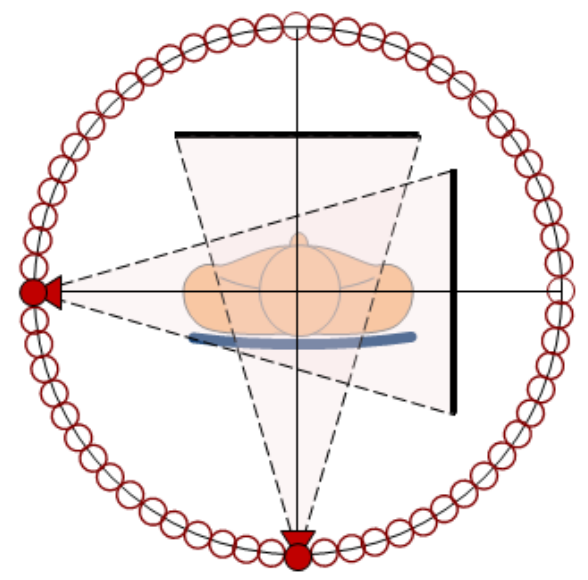

(c) Short orbit scan

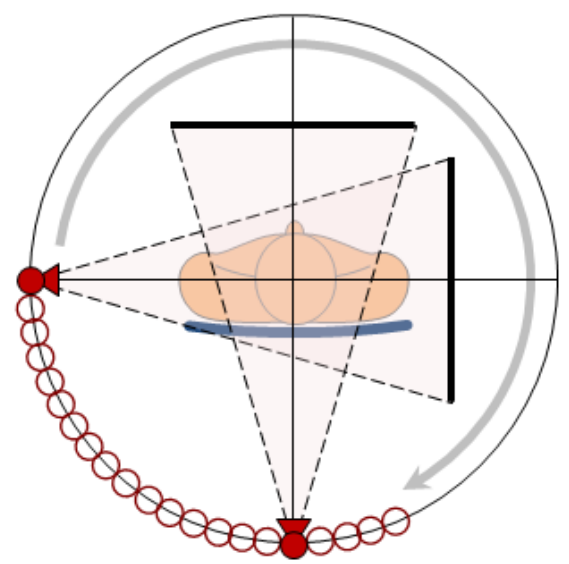

(b) Small views scan

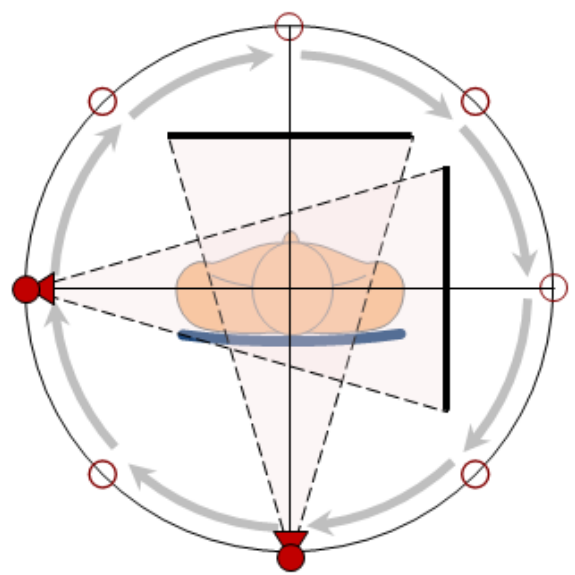

(d) Non-isocentric, small views and short orbit scan

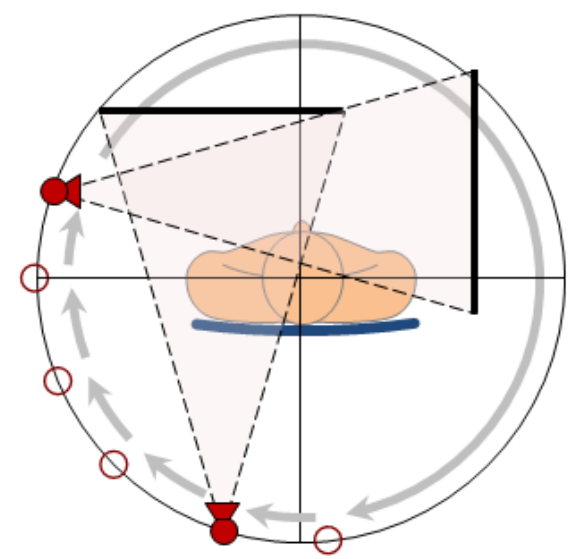

Figure 2: Sketch of different C-arm imaging configurations. (a) Full scan with severalhundred views, (b) scan with small number of views, (c) scan with short gantry rotation orbit, and (d) offset scan (non-isocenteric gantry) over small views and short rotation orbit. Area within detector FOV where the scanner gantry is located at the view angles of $0^{\circ}$ and $90^{\circ}$ is shown in different configurations for comparison. The configuration in (d) is the one considered in this study. 
(a)

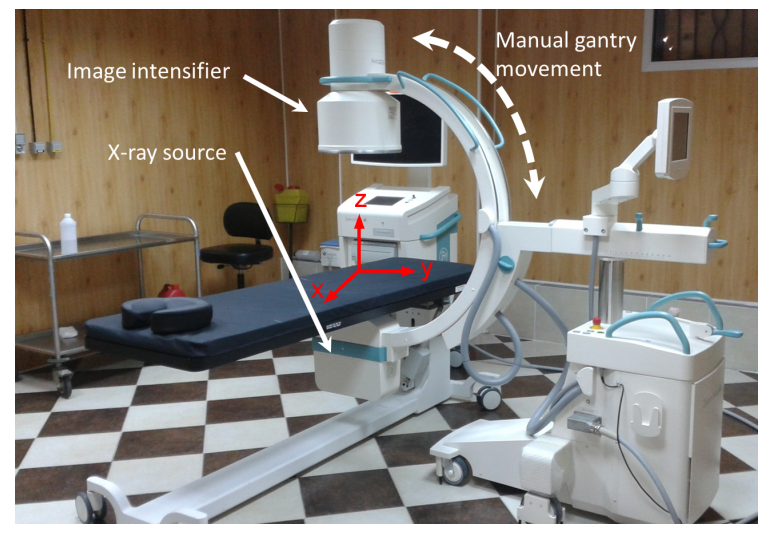

(b)

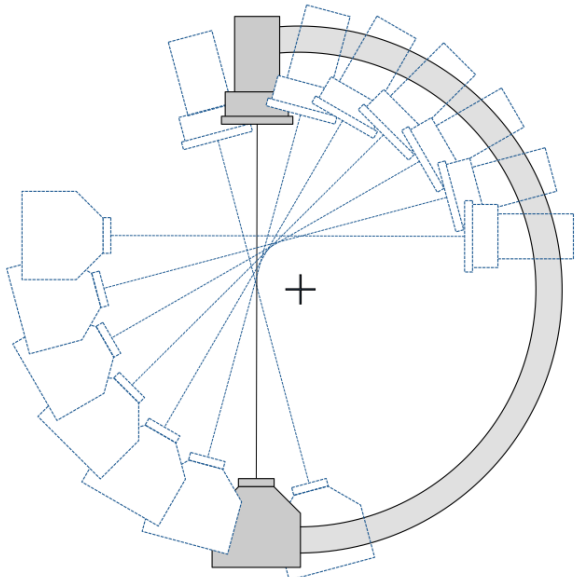

Figure 3: C-arm angiography system. (a) Photograph of the Ziehm Vision R scanner installed in SCUSH with gantry position of $\theta=90^{\circ}$. (b) Sketch of gantry positions for Carm scanner with non-isocentric geometry. Initial position with solid line is at the position angle of $90^{\circ}$. Dashed lines represent the gantry positions at angles of $0^{\circ}, 15^{\circ}, 30^{\circ}, 45^{\circ}$, $60^{\circ}, 75^{\circ}$ and $105^{\circ}$. The scanner ioscenter (center of gantry rotation) is indicated with the "+" sign. 

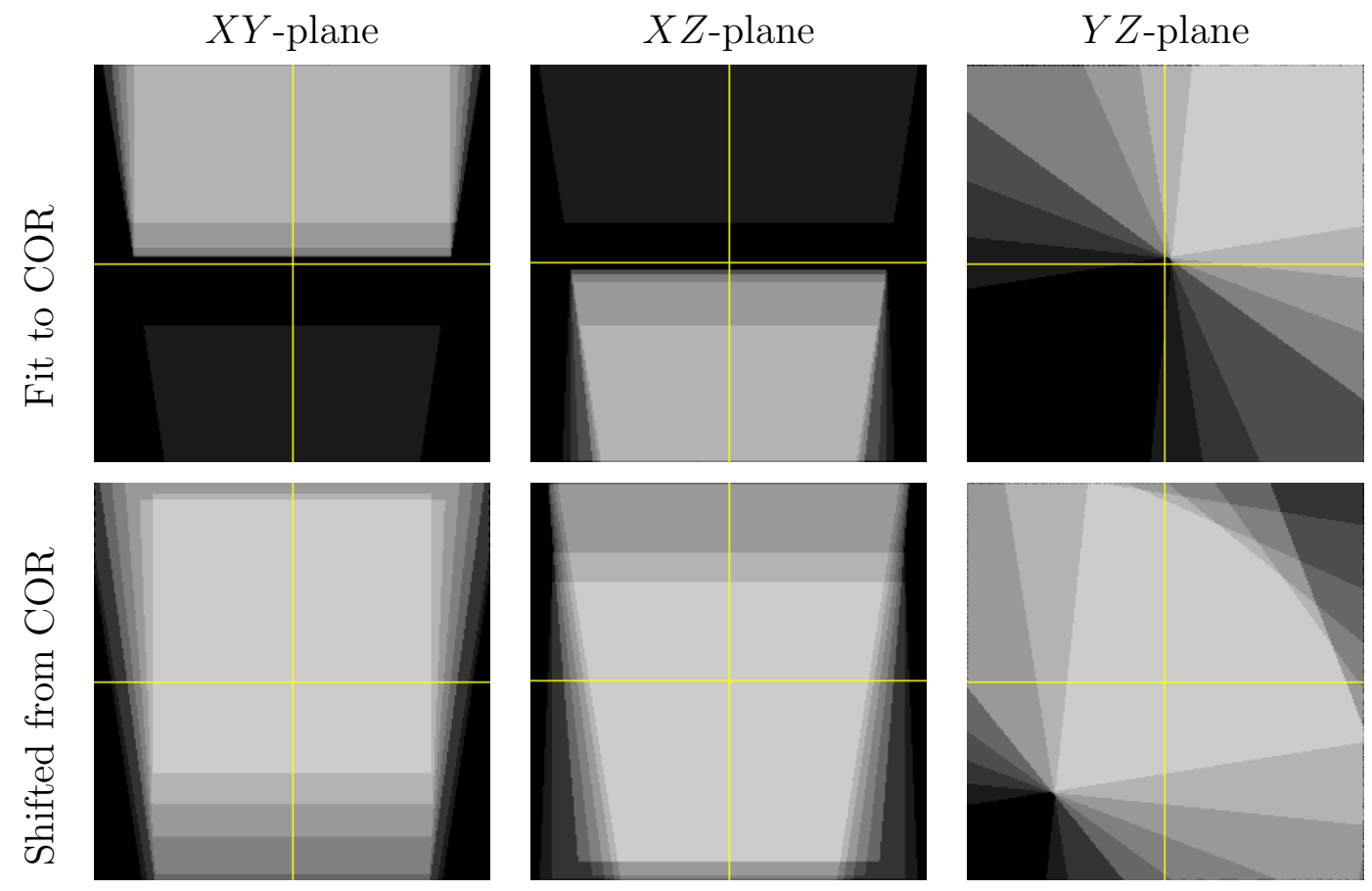

Figure 4: Orthogonal slices representing the number of projection rays passing through the image pixels. Data are acquired using commercial scanner geometry with 8 projection views indicated in Fig. 3(b). Top values are generated by locating the image center aligned to the scanner center of rotation. Bottom values are generated by shifting the image position in $\mathrm{YZ}$ plane (with value of $9.2 \mathrm{~cm}$ ) to maximize the number of rays passing through image pixels. Results shown in experimental studies below are computed using the shifted position shown in the bottom row. Grey scale is $[0,8]$. 
the commercial C-arm scanner used in this work in Fig. 3. To study the effect of non-isocentric geometry on data acquisition, we have counted the number of projection rays passing through each image pixel. We consider 8 projection views acquired from angular positions shown in Fig. 3(b). Simulation results are shown in Fig. 4. When the object is located at the center of rotation, large portion of object is likely located outside the FOV, however, this problem can be mitigated by shifting the object slightly in the YZ plane as shown in Fig. 4.

The combination of data limitation problems, described above, produces a challenging imaging system for which it is theoretically impossible to develop an accurate image reconstruction algorithm with stable solution. Alternatively, approximation methods are being used.

\section{Methods}

\subsection{Problem formulation}

In this section, we propose an image reconstruction algorithm devoted to the blood-vessel reconstruction problem from a limited number of projection data (possibly with limited angular coverage) as described in Section 2. Generally, image reconstruction from projection data can be formulated as solving a linear equation expressed as

$$
A \mathbf{x}=\mathbf{b}
$$

where $\mathbf{x}=\left(x_{1}, \ldots, x_{J}\right)^{\top}$ is a $J$-dimensional vector consisting of image pixel values to be reconstructed, $\mathbf{b}=\left(b_{1}, b_{2}, \ldots, b_{I}\right)^{\top}$ is an $I$-dimensional vector consisting of measured projection data, and $A=\left\{a_{i j}\right\}$ is an $I \times J$ matrix 
called the system matrix which relates $\mathbf{x}$ to $\mathbf{b}$. When the number of measured projection data $I$ is small, Eq. (1) becomes an underdetermined linear equation having many solutions because $I<J$. In such a case, it is common to formulate image reconstruction as the following constrained optimization problem.

$$
\min _{\mathbf{x}} f(\mathbf{x}) \text { subject to } A \mathbf{x}=\mathbf{b},
$$

where $f(\mathbf{x})$ is the cost function to pick up a reasonable image $\mathbf{x}$ with less artifacts from a set of feasible solutions satisfying Eq. (1). For this purpose, many cost functions have been proposed in the literature such as Gibbs prior [17], Total Variation (TV) [18], median root prior [19], and image sparsity

prior [4]. In this work, we use the image sparsity prior first proposed by Li et al. [4] for the blood-vessel reconstruction and investigated by others $[11,20,21]$, because the most significant feature of blood-vessel image is that the image itself has considerable sparsity in the sense that only a small number of pixels have non-zero values. In this prior, the cost function $f(\mathbf{x})$ is the $\ell_{1}$-norm of $\mathbf{x}$ defined as

$$
f(\mathbf{x})=\|\mathbf{x}\|_{1}^{1} \equiv \sum_{j=1}^{J}\left|x_{j}\right| .
$$

In the literature of CS (e.g. [22-24]), it is well-known that the value of Eq. (3) becomes small when $\mathbf{x}$ is sparse and its power to find a sparse solution is surprisingly strong in contrast to the ordinary $\ell_{2}$-norm of $\mathbf{x}$. This property allows accurate reconstruction even from a limited number of projection data as demonstrated in $[10,25]$ by using the $\ell_{1}$-norm of $\mathbf{x}$ as the cost function.

The optimization problem of Eq. (2) with Eq. (3) is named LASSO (least 
absolute shrinkage and selection operator) in the CS and machine learning fields, and there exist a variety of computational algorithms to find a solution [26]. However, these algorithms are not necessarily sufficient for the purpose of image reconstruction from the following point of view. Historically, in tomographic image reconstruction field, popular iterative algorithms employed in image reconstruction are ART (algebraic reconstruction technique), SART (simultaneous ART), and SIRT (simultaneous iterative reconstruction technique) [27]. These algorithms have attractive features such as simplicity of implementation and storage efficiency. Furthermore, it is known that ART can be converged very fast with few iterations by using some special data access order [28]. This behavior is due to the increase of the orthogonality among projections at each iteration and is confirmed by several later studies (e.g. [4, 29-31]). Therefore, there exists a strong demand in developing a class of iterative algorithms having the similar structure to ART or using ART as a major building block. For the problem of Eq. (2) with Eq. (3), such an iterative algorithm has been already proposed and implemented by Li et al. [4]. In this paper, we propose and implement an alternative iterative algorithm derived from the optimization method called ADMM which is attracting a lot of attention recently.

\subsection{Brief review of $A D M M$}

We begin by a brief review of ADMM in the form appropriate for our algorithm derivation. We refer [32] for a rigorous and detailed review of ADMM. Let us consider the constrained optimization problem formulated as

$$
\min _{(\mathbf{x}, \mathbf{z})} f(\mathbf{x})+g(\mathbf{z}) \text { subject to } A \mathbf{x}+B \mathbf{z}=\mathbf{c},
$$


where $\mathbf{x}=\left(x_{1}, x_{2}, \ldots, x_{J_{1}}\right)^{\top}$ and $\mathbf{z}=\left(z_{1}, z_{2}, \ldots, z_{J_{2}}\right)^{\top}$ are $J_{1}$-dimensional vectors and $J_{2}$ dimensional vector, respectively, c is an $I$-dimensional vector, $A=\left\{a_{i j}\right\}$ and $B=\left\{b_{i j}\right\}$ are $I \times J_{1}$ matrix and $I \times J_{2}$ matrix, respectively, and we assume that both $f(\mathbf{x})$ and $g(\mathbf{z})$ are (possibly non-differentiable ) convex functions. In Eq. (4), the two variables $\mathbf{x}$ and $\mathbf{z}$ are coupled only through the linear constraint $A \mathbf{x}+B \mathbf{z}=\mathbf{c}$. In ADMM, the iterative algorithm to solve this problem is constructed according to the following procedure. First, we define the augmented Lagrangian function corresponding to the problem of Eq. (4) by

$$
L_{\rho}(\mathbf{x}, \mathbf{z}, \boldsymbol{\lambda})=f(\mathbf{x})+g(\mathbf{z})+\boldsymbol{\lambda}^{\top}(A \mathbf{x}+B \mathbf{z}-\mathbf{c})+\frac{\rho}{2}\|A \mathbf{x}+B \mathbf{z}-\mathbf{c}\|_{2}^{2},
$$

where $\boldsymbol{\lambda}$ is an $I$-dimensional vector called the dual vector or the Lagrange multiplier vector and $\rho>0$ is the weighting parameter of the second-order penalty term. Using Eq. (5), the iterative algorithm of ADMM is expressed as the following three-step procedure (with $k$ as the iteration number).

[x minimization]

$$
\mathbf{x}^{(k+1)}=\arg \min _{\mathbf{x}} L_{\rho}\left(\mathbf{x}, \mathbf{z}^{(k)}, \boldsymbol{\lambda}^{(k)}\right)
$$

[z minimization]

$$
\mathbf{z}^{(k+1)}=\arg \min _{\mathbf{z}} L_{\rho}\left(\mathbf{x}^{(k+1)}, \mathbf{z}, \boldsymbol{\lambda}^{(k)}\right)
$$

[Multiplier $\boldsymbol{\lambda}$ update]

$$
\boldsymbol{\lambda}^{(k+1)}=\boldsymbol{\lambda}^{(k)}+\rho\left(A \mathbf{x}^{(k+1)}+B \mathbf{z}^{(k+1)}-\mathbf{c}\right)
$$


In each iteration, this iterative algorithm minimizes $L_{\rho}(\mathbf{x}, \mathbf{z}, \boldsymbol{\lambda})$ with respect to $\mathbf{x}$ and $\mathbf{z}$ alternately followed by updating the multiplier $\boldsymbol{\lambda}$ in the ascent direction of $L_{\rho}(\mathbf{x}, \mathbf{z}, \boldsymbol{\lambda})$. The attractive feature of ADMM is that the minimization of $f(\mathbf{x})+g(\mathbf{z})$ can be split into the sequence of two separate minimizations with respect to $f(\mathbf{x})$ and $g(\mathbf{z})$. This is a powerful framework in many instances of optimization including the constrained optimization and the optimization where at least either $f(\mathbf{x})$ and $g(\mathbf{z})$ is non-differentiable.

Finally, we remark how ADMM differs from the classical method of multipliers to solve the constrained optimization. In the classical method of multipliers, instead of the augmented Lagrangian $L_{\rho}(\mathbf{x}, \mathbf{z}, \boldsymbol{\lambda})$, the ordinary Lagrangian function defined by the following equation is used.

$$
L(\mathbf{x}, \mathbf{z}, \boldsymbol{\lambda})=f(\mathbf{x})+g(\mathbf{z})+\boldsymbol{\lambda}^{\top}(A \mathbf{x}+B \mathbf{z}-\mathbf{c})
$$

The principle difference between Eq. (5) and Eq. (9) is that the second-order penalty term is missing in Eq. (9). Without the second-order term, the minimization problem in Eq. (6) or Eq. (7) may not admit a unique solution in the case where $f(\mathbf{x})$ or $g(\mathbf{z})$ is not strongly convex (such as the case where $f(\mathbf{x})$ or $g(\mathbf{z})$ is an characteristic function or a non-strongly convex function). In such a case, the classical method of multipliers fails in generating a valid iterates of $\mathbf{x}$ and $\mathbf{z}$, but ADMM is still valid thanks to the existence of strongly convex additional second-order penalty term. As described later, in our problem of Eq. (2) with Eq. (3), $f(\mathbf{x})$ is the $\ell_{1}$-norm of $\mathbf{x}$ and $g(\mathbf{z})$ is the characteristic function of affine subspace $A \mathbf{z}=\mathbf{b}$, both of which are not strongly convex. For this reason, using ADMM instead of the method of multipliers is absolutely necessary to derive the proposed algorithm. 


\subsection{Derivation of proposed algorithm}

Next, we explain how ADMM is used to derive an iterative algorithm to solve the problem of Eq. (2) with the cost function given by Eq. (3). First, by introducing the new variable $\mathbf{z}$, this problem can be reformulated into the following form.

$$
\min _{(\mathbf{x}, \mathbf{z})} f(\mathbf{x})+g(\mathbf{z}) \text { subject to } \mathbf{x}-\mathbf{z}=0,
$$

where $f(\mathbf{x})$ and $g(\mathbf{z})$ are defined by

$$
\begin{gathered}
f(\mathbf{x})=\|\mathbf{x}\|_{1}^{1} \\
g(\mathbf{z})= \begin{cases}0 & \text { (if } A \mathbf{z}=\mathbf{b}) \\
\infty & \text { (otherwise) }\end{cases}
\end{gathered}
$$

We note that $g(\mathbf{z})$ is the characteristic function corresponding to the affine subspace $A \mathbf{z}=\mathbf{b}$ [33]. It can be easily checked that the solution to Eqs. (10)(12) coincides with the solution to Eq. (2) with Eq. (3). Furthermore, Eqs. (10)-(12) is a special case of Eq. (4), i.e. $J_{1}=J_{2}=J, A=E, B=-E$, and $\mathbf{c}=0$ in Eq. (4) ( $E$ is an identity matrix), to which ADMM can be applied to construct an iterative algorithm. Applying ADMM to the problem of Eqs. (10)-(12), we obtain the following three-step iterative algorithm.

$$
\begin{array}{r}
\mathbf{x}^{(k+1)}=\arg \min _{\mathbf{x}}\left[f(\mathbf{x})+\boldsymbol{\lambda}^{(k)^{\top}}\left(\mathbf{x}-\mathbf{z}^{(k)}\right)+\frac{\rho}{2}\left\|\mathbf{x}-\mathbf{z}^{(k)}\right\|_{2}^{2}\right] \\
\mathbf{z}^{(k+1)}=\arg \min _{\mathbf{z}}\left[g(\mathbf{z})+\boldsymbol{\lambda}^{(k)^{\top}}\left(\mathbf{x}^{(k+1)}-\mathbf{z}\right)+\frac{\rho}{2}\left\|\mathbf{x}^{(k+1)}-\mathbf{z}\right\|_{2}^{2}\right]
\end{array}
$$




$$
\boldsymbol{\lambda}^{(k+1)}=\boldsymbol{\lambda}^{(k)}+\rho\left(\mathbf{x}^{(k+1)}-\mathbf{z}^{(k+1)}\right)
$$

The algorithm expressed by Eqs. (13)-(15) still does not have a concrete implementable form. So, we further simplify Eqs. (13)-(15) below. First, since the cost function in Eq. (13) is the sum of $\ell_{1}$-norm of $\mathbf{x}$ and the quadratic term, which frequently appears in the CS literature, the $\mathbf{x}$ minimization in Eq. (13) can be solved in closed form. The solution is given by

$$
\mathbf{x}^{(k+1)}=\operatorname{SoftThre}\left(\mathbf{z}^{(k)}-\boldsymbol{\lambda}^{(k)} / \rho\right),
$$

where the operator SoftThre(.) is defined by

$$
\begin{gathered}
\operatorname{SoftThre}(\mathbf{y})=\left(T h\left(y_{1}\right), T h\left(y_{2}\right), \ldots, T h\left(y_{J}\right)\right)^{\top} \\
T h(y)= \begin{cases}y-1 / \rho & (\text { if } y>1 / \rho) \\
y+1 / \rho & (\text { if } y<-1 / \rho) . \\
0 & \text { (otherwise) }\end{cases}
\end{gathered}
$$

We note that SoftThre(.) is the so-called soft-thresholding operator used in the CS field [34], which sets vector components having small absolute values to zeros and moving other components toward zeros by an amount of $1 / \rho$. Next, we describe how to solve the $\mathbf{z}$ minimization of Eq. (14). Since $g(\mathbf{z})$ is the characteristic function corresponding to $A \mathbf{z}=\mathbf{b}$, the solution $\mathbf{z}^{(k+1)}$ to this problem can be achieved by solving the following linearly constrained optimization [32].

$$
\min _{\mathbf{z}}\left\|\mathbf{z}-\left(\mathbf{x}^{(k+1)}+\boldsymbol{\lambda}^{(k)} / \rho\right)\right\|_{2}^{2} \text { subject to } A \mathbf{z}=\mathbf{b}
$$


Since Eq. (18) is the standard Euclidean distance minimization subject to a linear equality constraint, a variety of methods in the linear algebra field can be used to solve it. However, the problem size is so large that we need to adopt a storage efficient method. In this work, we use ART iterative method with the initial vector $\mathbf{x}^{(k+1)}+\boldsymbol{\lambda}^{(k)} / \rho$, which is known to be efficient and easy to implement. The detailed algorithm for the $\mathbf{z}$ minimization using ART can be summarized in the following form.

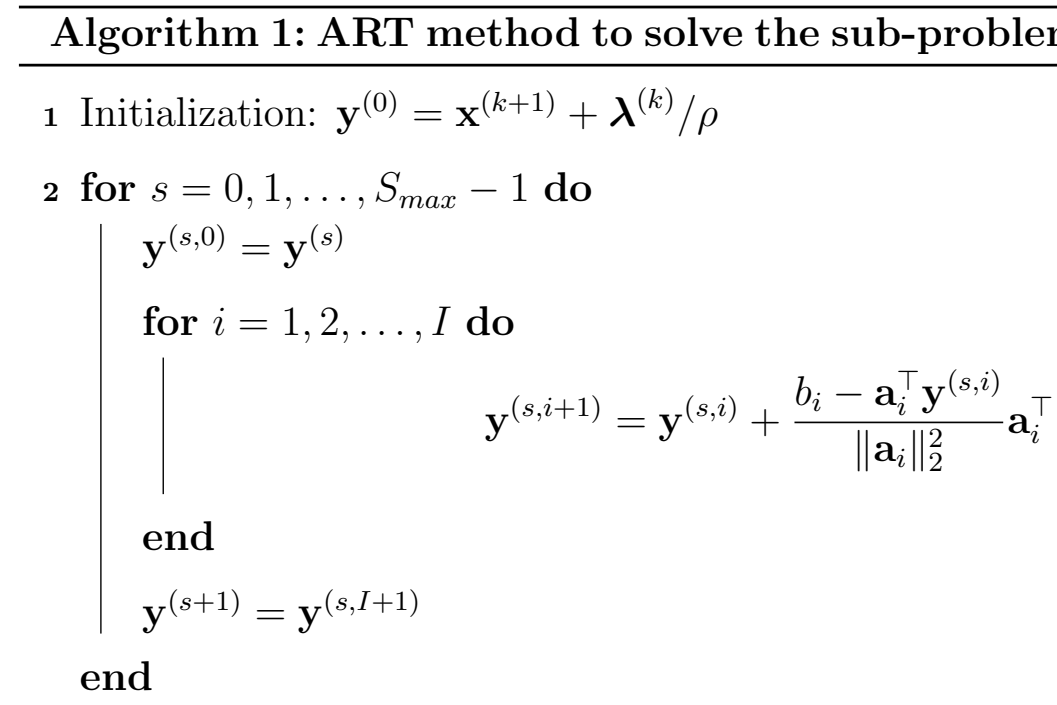

3 Solution $\mathbf{z}^{*}=\mathbf{y}^{\left(S_{\max }\right)}$

where $\mathbf{a}_{i}=\left(a_{i 1}, a_{i 2}, \ldots, a_{i n}\right)^{\top}$ is the $\mathrm{i}$ - $t h$ row of system matrix $A$ and $s$ is the iteration number. With respect to the convergence of the above ART iteration, the following theorem is well-known $[35,36]$. We write it without the proof.

Theorem 1. Assume that there exist $\mathbf{z}$ satisfying the linear equation $A \mathbf{z}=$ $\mathbf{b}$, i.e. $A \mathbf{z}=\mathbf{b}$ is feasible. Then, the solution to problem of Eq. (18) is unique and the above ART method converges to $\mathbf{z}^{*}=\mathbf{y}^{(\infty)}$ which minimizes the distance $\left\|\mathbf{z}-\mathbf{y}^{(0)}\right\|_{2}^{2}$ with $\mathbf{y}^{(0)}=\mathbf{x}^{(k+1)}+\boldsymbol{\lambda}^{(k)} / \rho$ among all $\mathbf{z}$ satisfying 
$A \mathbf{z}=\mathbf{b}$.

For convenience, we express the above ART iteration as

$$
\mathbf{z}^{*}=\operatorname{ART}\left(\mathbf{x}^{(k+1)}+\boldsymbol{\lambda}^{(k)} / \rho\right)
$$

In tomographic image reconstruction, the so-called non-negativity constraint $\mathbf{x} \geqq 0$ is often used, which dramatically improves image quality in some applications. We expect that the blood-vessel reconstruction is one of such applications, because values of most background image pixels are zeros or close to zeros. The non-negativity constraint can be incorporated into the proposed algorithm by modifying the algorithm derivation described in Section 3.3 as follows. First, by incorporating $\mathbf{x} \geqq 0$ into the cost function $f(\mathbf{x})$, the original problem Eq. (3) is modified to

$$
f(\mathbf{x})=\left\{\begin{array}{ll}
\|\mathbf{x}\|_{1}^{1} & (\text { if } \mathbf{x} \geq \mathbf{0}) \\
\infty & \text { (otherwise) }
\end{array} .\right.
$$

The infinite value in Eq. (20) is added to exclude the negative vales of $\mathbf{x}$ from the set of feasible solutions of the minimization problem. Then, we can follow the same algorithm derivation as in Section 3.3. The only necessary change appears in solving the sub-problem of Eq. (13), in which we need to take $\mathbf{x} \geqq 0$ into account. The resulting soft-thresholding operation under $\mathbf{x} \geqq 0$ is obtained as

$$
\mathbf{x}^{(k+1)}=\operatorname{SoftThre}\left(\mathbf{z}^{(k)}-\boldsymbol{\lambda}^{(k)} / \rho\right),
$$

$$
\operatorname{SoftThre}(\mathbf{y})=\left(T h\left(y_{1}\right), T h\left(y_{2}\right), \ldots, T h\left(y_{J}\right)\right)^{\top}
$$


(a)

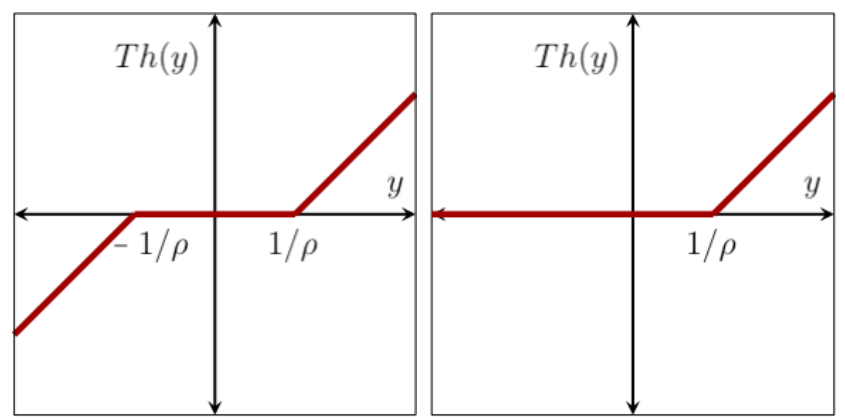

Figure 5: Illustration of soft-thresholding functions corresponding to (a) Eq. (17) and (b) Eq. (21).

$$
T h(y)=\left\{\begin{array}{ll}
y-1 / \rho & (\text { if } y>1 / \rho) \\
0 & \text { (otherwise) }
\end{array} .\right.
$$

The comparison between Eq. (17) and Eq. (21) shows that all negative values must be set to zeros together with small positive values in the SoftThre(.) operation when using the non-negativity constraint. The difference between the soft-thresholding in Eq. (17) and that in Eq. (21) is shown in Fig. 5. This is the only change required to impose the non-negativity constraint, which seems to be minimum. Finally, we note that it is also possible to incorporate $\mathbf{z} \geqq 0$ into the $g(\mathbf{z})$ term instead of incorporating $\mathbf{x} \geqq 0$ into the $f(\mathbf{x})$ term. However, in this case, a large modification is required in the inner ART iteration (we omit the details). So, we believe that applying ADMM to the formulation of Eq. (20) is the better method of choice to handle the non-negativity constraint. 


\subsection{Summary of proposed algorithm}

Basically, the proposed algorithm is given by Eqs. (13)-(15), in which we showed that the $\mathbf{x}$ minimization in Eq. (13) reduces to the soft-thresholding and the $\mathbf{z}$ minimization in Eq. (14) can be performed using the inner ART iteration. The final algorithm can be summarized in the following form.

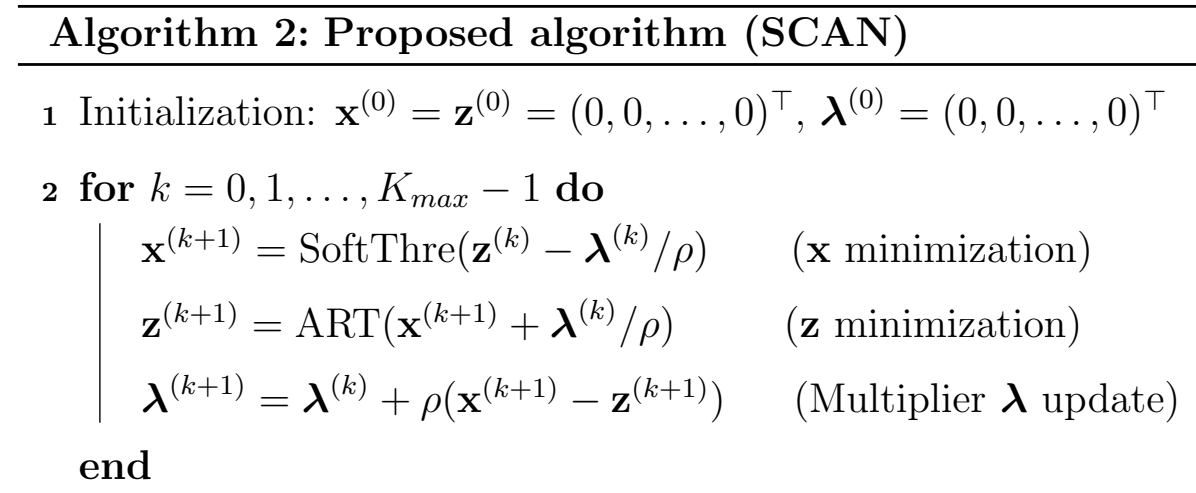

3 Solution $\mathbf{z}^{*}=\mathbf{z}^{\left(K_{\max }\right)}$

where we remark that the operations SoftThre(.) and ART(.) are specified in Section 3.3.

The SCAN algorithm possesses a nice structure in the sense that its major operations consist of the soft thresholding and the ART iteration, both of which are popular in recent research of tomographic image reconstruction. One drawback of the proposed algorithm is that it has a double-loop (second-order) structure so that the convergence to the true solution may not be guaranteed if the inner ART iteration is terminated after only a small number of iterations. Of course, to save computational time, we would like to terminate the inner iteration as early as possible. Therefore, it is a practically important issue to investigate how many iterations are necessary in the inner ART iteration. In our simulation studies, by using the special data access order of ART proposed in [28], we observed that only a single inner iteration of 
(a) 3D phantom

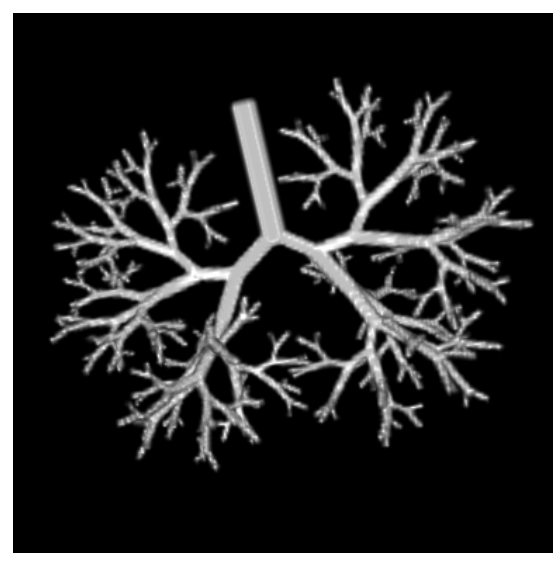

(b) Central 2D slice

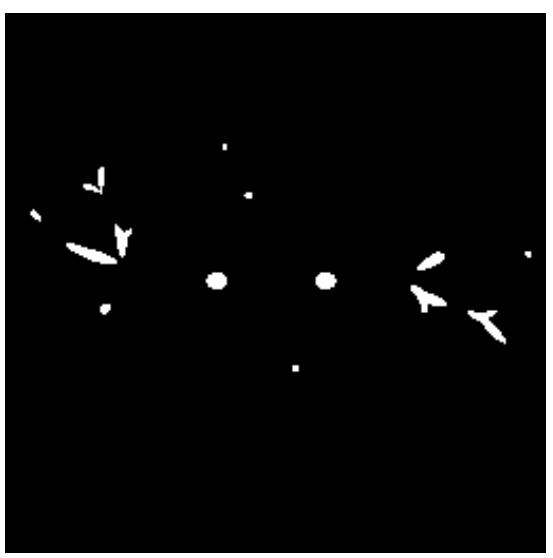

Figure 6: (a) Volume rendering of the digital phantom object and (b) central 2D crosssectional slice.

ART is sufficient to obtain satisfactory reconstructed images (i.e. $S_{\max }=1$ ). Therefore, the proposed algorithm possesses fewer parameters to adjust in practical implementation. However, at present, we do not have a theoretical convergence proof of the proposed algorithm implemented with a finite number of inner ART iterations. Finally, we write a convergence property of the proposed algorithm proved from the known convergence result of ADMM $[32]$.

Theorem 2. Assume that the original problem of Eq. (2) with Eq. (3) has a unique solution. Then, under the assumption that the number of inner ART iteration is infinite, the proposed algorithm converges to $\mathbf{z}^{*}=\mathbf{z}^{(\infty)}$ which solves the original problem of Eq. (2) with Eq. (3). 


\section{Results and Discussion}

\subsection{Simulated data}

A computer simulation study was performed to evaluate the proposed method. A digital phantom (FORBILD lung phantom ${ }^{2}$ ) representing a vessel-like sparse structure, shown in Fig. 6, was used in this study. This phantom was originally created to represent lung bronchial airways. However, it is also used in several studies to represent blood-vessels structures $[4,11]$. The phantom volume was of $256 \times 256 \times 256$ pixels with binary attenuation formats (i.e. vessel pixels are 1's and background pixels are 0's). Mono-energetic cone-beam projection data were simulated at 6, 8, 12 and 24 views with rotation angle spacing of $20^{\circ}, 15^{\circ}, 10^{\circ}$ and $5^{\circ}$, respectively, over the gantry rotation arc of $115^{\circ}$. The scanner geometry parameters were set to real parameters of the Ziehm Vision R C-arm scanner detailed in Section 2 with tube voltage of $100 \mathrm{kV}$. For simplicity, we did not consider scatter and noise effects. Due to the scanner geometry settings, the scanner FOV that is visible in all view angles, is rather small. Consequently, all projection views suffer from the data truncation.

To demonstrate the effect of offset scan due to the scanner non-isocenteric geometry, we show the projection images acquired from different view angles $\theta$ in the cases of isocenteric and non-isocenteric configurations in Fig. 7. System configuration parameters for isocentric and non-isocentric experiments are shown in table 1. It is clear from Fig. 7, that for the isocentric scanner geometry, the phantom is located almost completely inside the scan-

\footnotetext{
${ }^{2}$ http://www.imp.uni-erlangen.de/forbild/english/results/index.htm
} 

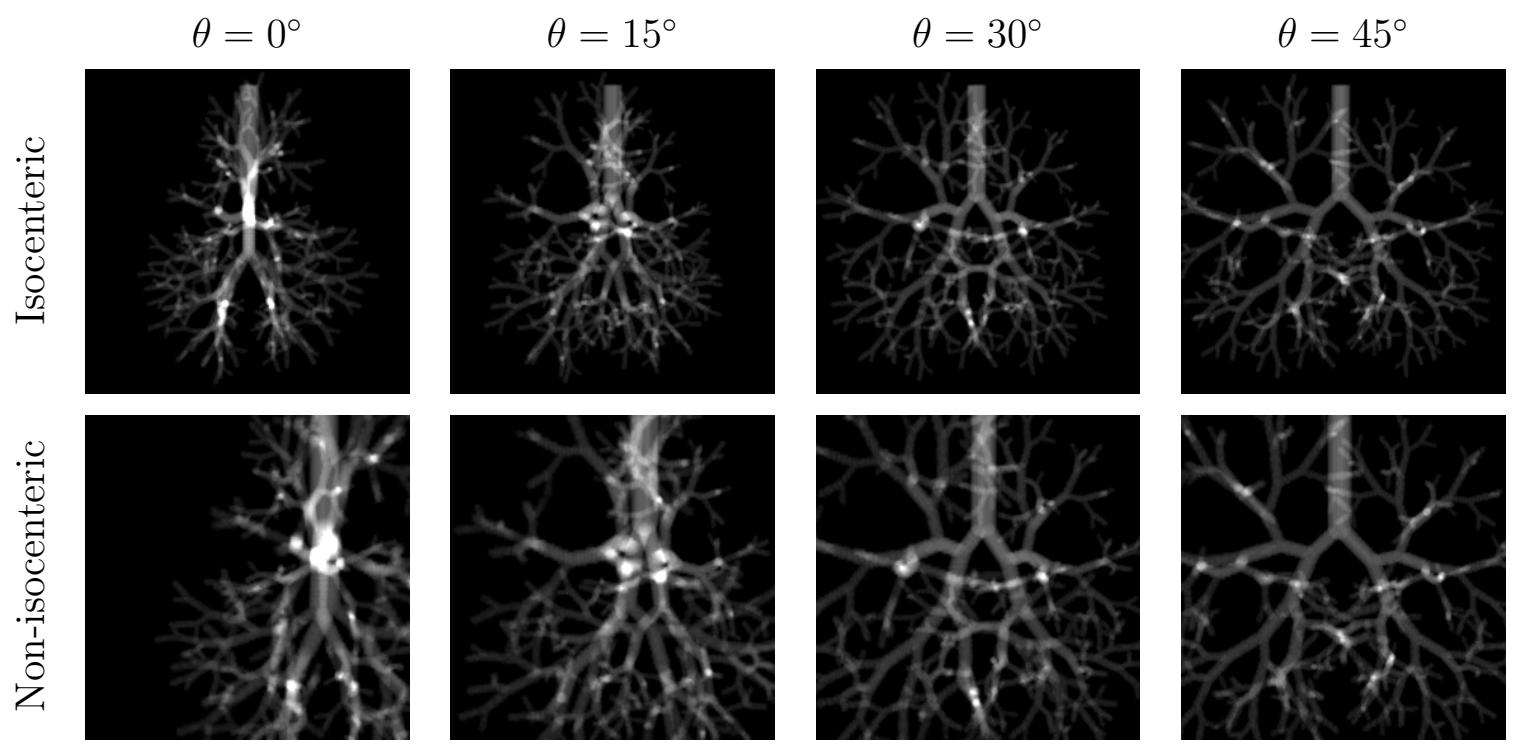

$$
\theta=60^{\circ}
$$
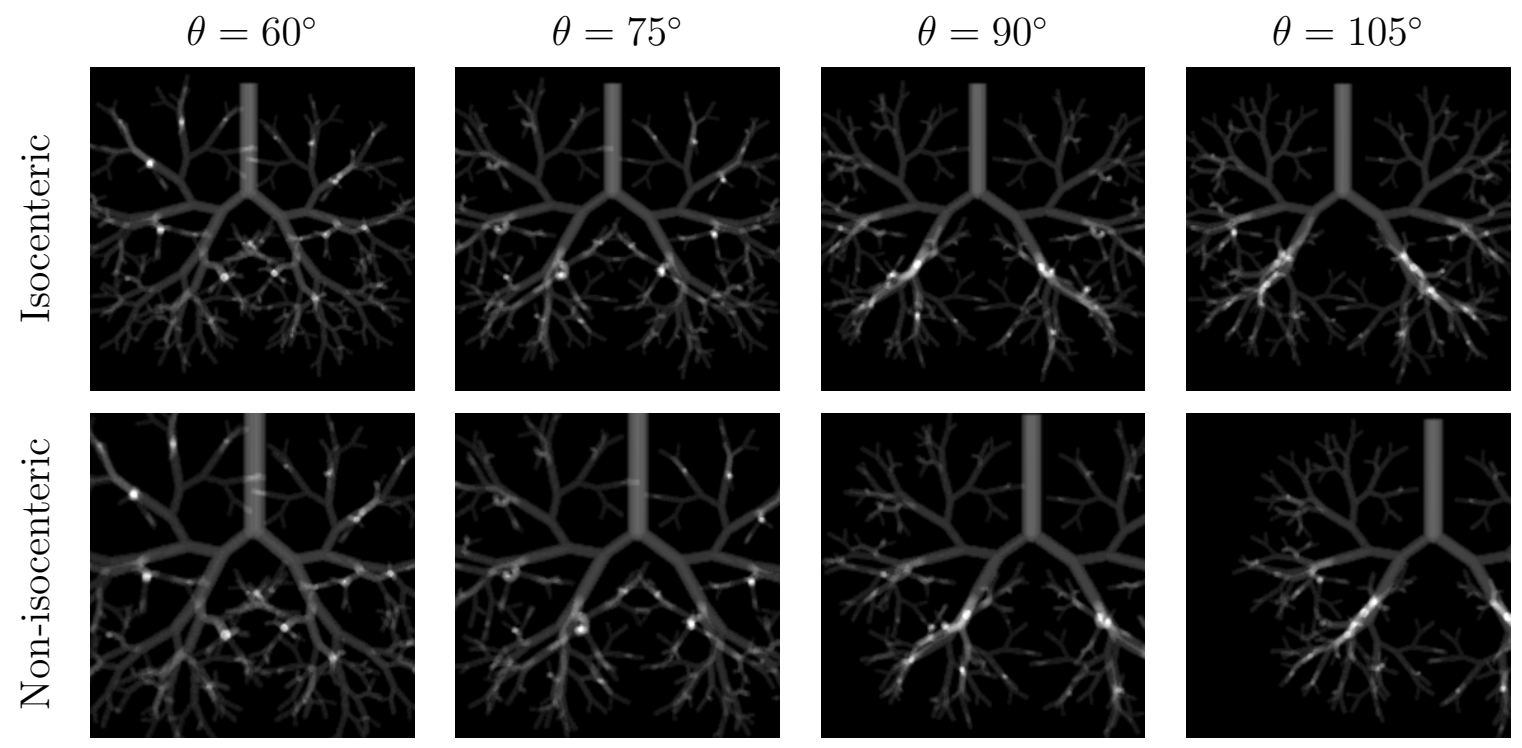

Figure 7: Projection images of the digital phantom corresponding to different view angles $\theta$ with the isocenteric and non-isocenteric scanner configurations. 
Table 1: Simulation parameters used to compute projection data shown in Fig. 7.

\begin{tabular}{llcc}
\hline Parameter & Description & \multicolumn{2}{c}{ Value $(\mathrm{cm})$} \\
& & isocenteric & non-isocenteric \\
\hline SDD & Source to detector distance & 97.0 & 97.0 \\
DCOR & Detector to center-of-rotation (COR) & 18.0 & 36.43 \\
DO & Detector offset & $\mathrm{N} / \mathrm{A}$ & 13.0 \\
OCOR & Object to COR $(\mathrm{x}, \mathrm{y}, \mathrm{z})$ & $(0,0,0)$ & $(0,9.2,9.2)$ \\
\hline
\end{tabular}

ner FOV. However, the projection data measured using the non-isocentric geometry suffer from data truncation with different degrees. Moreover, the object appears in different magnification scales in each view angle as the distance between the scanned object and the scanner detector varies dependent on the view angle. The data incompleteness in each projection view produces a challenge in image reconstruction in addition to that of the small number of projection views.

The SCAN algorithm was implemented with parameters of $\rho=20, S_{\max }=$ 1 and $K_{\max }=20$ to reconstruct $3 \mathrm{D}$ volumes from $6,8,12$ and 24 projection views. We consider both isocenteric and non-isocenteric scanner geometry. Reconstruction results are shown in Fig. 8 and the corresponding transverse cross-sectional slices are shown in Fig. 9. We also calculate the relative root mean square error (RRME) as similarity-based metric. RRME is calculated using the following formula:

$$
\operatorname{RRME}(\mathbf{x})=\sqrt{\sum_{j=1}^{J}\left(x_{j}-\tilde{x}_{j}\right)^{2} / \sum_{j=1}^{J}\left(\tilde{x}_{j}\right)^{2}}
$$

where $\tilde{\mathbf{x}}=\left(\tilde{x}_{1}, \ldots, \tilde{x}_{J}\right)$ is the true phantom. For convenience, RRME value is 
(a) 6 views
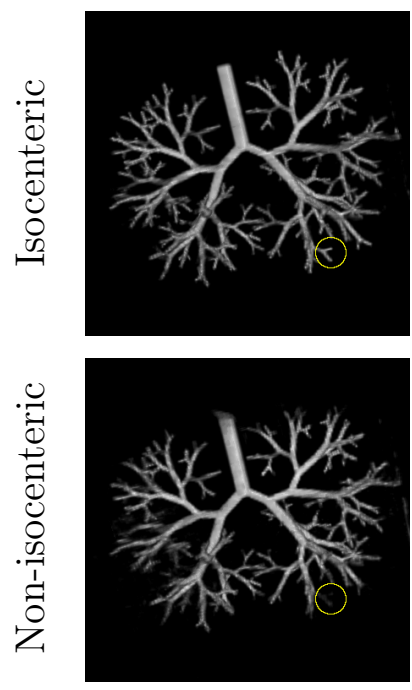

(b) 8 views
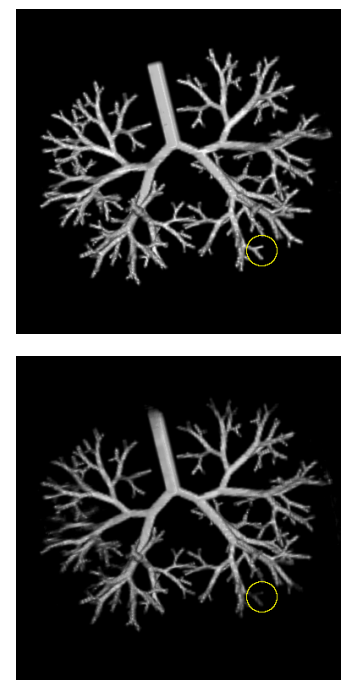

(c) 12 views
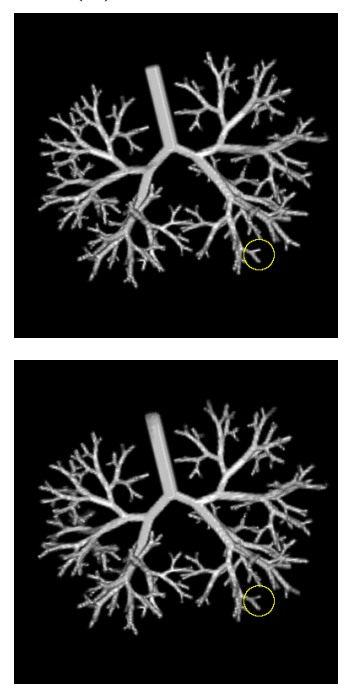

(d) 24 views
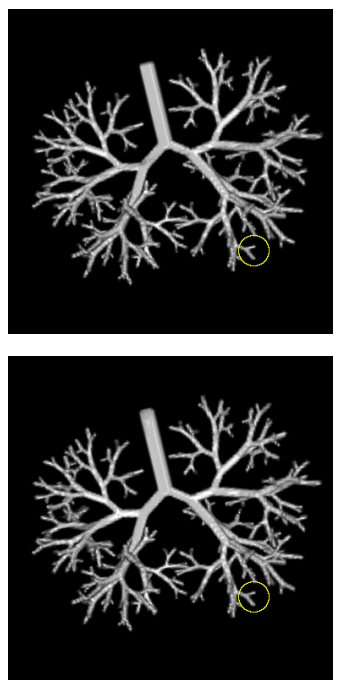

Figure 8: Three-dimensional volume rendering of SCAN reconstruction with different projection views using true phantom shown in Fig. (6). Top and bottom rows are corresponding to isocenteric and non-isocentric scanner configurations, respectively. Mark circle indicates peripheral vessel reconstructed in different quality corresponding to the number of projection views. 
shown at the top left corner of each image. Supplementary materials associated to this paper includes a $360^{\circ}$ rotation of the rendered $3 \mathrm{D}$ reconstructed image shown in Fig. 8(d) for non-isocenteric geometry. From these results, it can be observed that the proposed method can reconstruct an appropriate image even with only 8 projection views. Appropriate image can be defined as image in which vessel branches and connections can be defined correctly to guide road-mapping of the catheter. However, it is obvious that blood-vessels with small size located in peripheral regions are difficult to be observed when the number of projection views is highly down-sampled. As shown in Fig. 8, small vessels marked with circle cannot be observed with 6 views and visual observation is gradually improved with higher number of views. To compare the proposed algorithm with the conventional ART algorithm, cross-sectional slices obtained from images reconstruction using same number of iterations (20 iterations) are shown in Fig. 9. Due to strong streak artifacts in ART images, it was impossible to achieve volume rendered images to be compared with those in Fig. 8. While the images obtained by the proposed method are very close to the true object, even for small size vessels, the ART reconstruction suffers from strong streak artifacts tangent to the boundaries of vessels. Comparison quantitative results using RRME as quality measure is shown in Fig. 10. The 3D reconstruction using ART and SCAN algorithms were implemented in C programming language and evaluated using PC (Intel (R) Core (TM) i7 @ 2.4 GHz, Memory: 8.0 GB) and single iteration computation time is shown in Table 2. It is clear that time difference is small as the time used for thresholding operation and multiplier update in SCAN algorithm is rather small compared to ART image update step. 
Table 2: Computation time in seconds for single iteration of 3D reconstruction using ART and SCAN algorithms.

\begin{tabular}{ccc}
\hline \# of views & $\begin{array}{c}\text { Algorithm } 1(\mathrm{ART}) \\
S_{\max }=1\end{array}$ & $\begin{array}{c}\text { Algorithm } 2(\mathrm{SCAN}) \\
K_{\max }=1 \& S_{\max }=1\end{array}$ \\
\hline 6 & 14.1 & 15.0 \\
8 & 18.3 & 19.1 \\
12 & 27.3 & 27.9 \\
24 & 52.8 & 53.1 \\
\hline
\end{tabular}
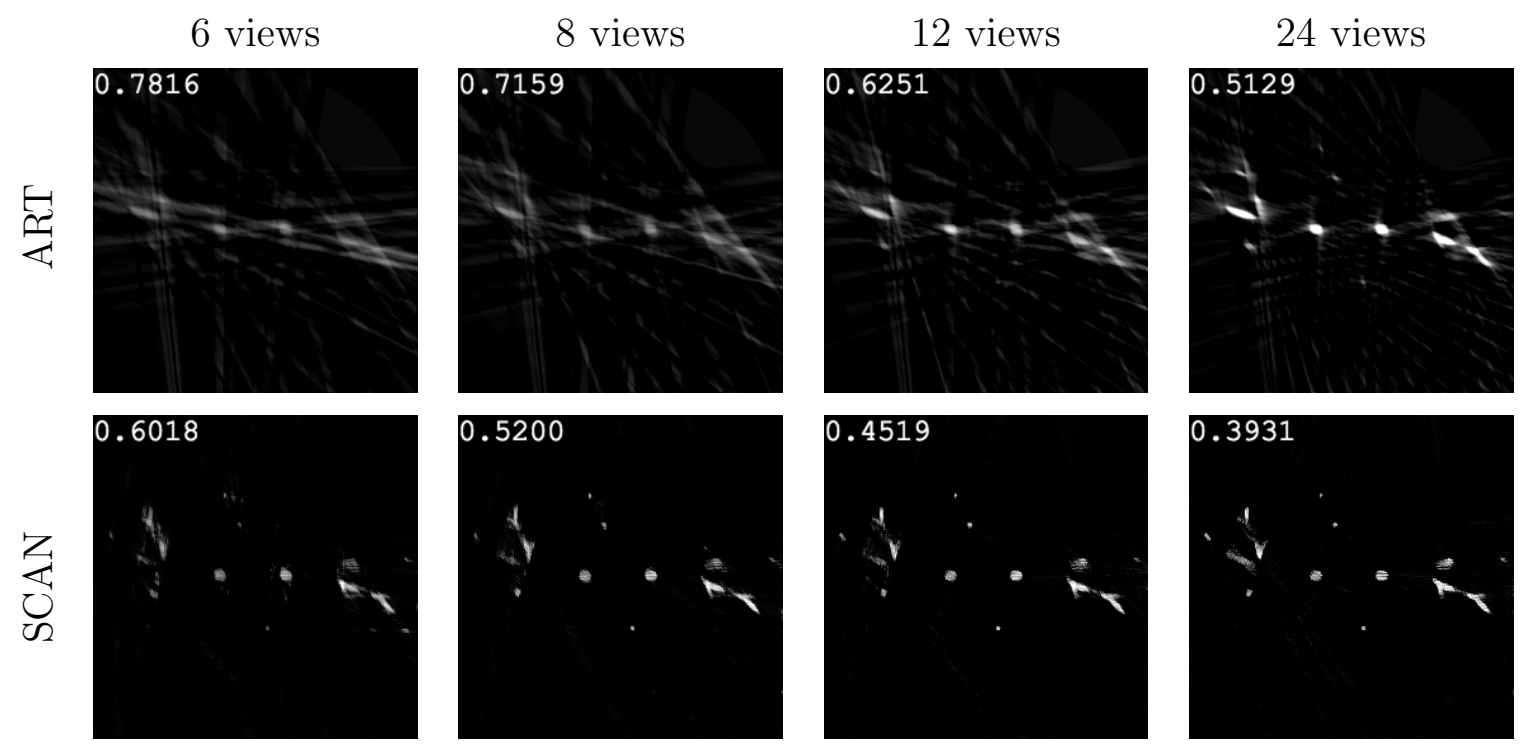

Figure 9: Single corresponding cross-sectional slices of reconstructed images from different projection views using $\operatorname{ART}\left(S_{\max }=20\right)$ and $\operatorname{SCAN}\left(K_{\max }=20, S_{\max }=1\right.$ and $\left.\rho=20\right)$. Bottom row is a $2 \mathrm{D}$ slice of the $3 \mathrm{D}$ images shown in Fig. 8 with non-isocenteric geometry. RRME value is shown in the top left corner of each image. 
It is important to study the performance of the SCAN algorithm with different image reconstruction parameters such as $K_{\max }, S_{\max }$ and $\rho$. A comparison study was used to demonstrate the effect of each parameter to the quality of the reconstructed image. We consider 24 projection views obtained using non-isocenteric scanner geometry. Images are reconstructed with different values of $K_{\max }=\{1,5,10,20\}, S_{\max }=\{1,5,10,20\}$ and $\rho=$ $\{0.1,1.0,10.0,20.0,100.0\}$. First, we set $\rho=20$ and reconstruct images with different values of $K_{\max }$ and $S_{\max }$. Central cross-sectional slices are shown in Fig. 11. From these results, we can observe that increasing the value of $S_{\max }$ has relatively small contribution to the image quality compared to the value of $K_{\max }$. This observation is useful in practical implementation as we can set the value of $S_{\max }$ to single iteration. Second, we set $S_{\max }=1$ and obtain results with variant values of $K_{\max }$ and $\rho$. Achieved comparison results are shown in Fig. 12. It is observed that the value of $\rho$ is important factor in image quality. For example, image quality improves very slowly when we iterate with $\rho=0.1$. However, with higher value of weighting parameter of the second-order penalty (i.e. $\rho=10.0$ ), we observe a relatively large improvement in image quality after only few iterations $\left(K_{\max }=5\right)$.

\subsection{Real data}

A real data experiment was performed for the assessment of the proposed method. A metal wire was used to simulate human segmental hepatic arterial system imaged with the DSA approach. The metal object is a $6 \mathrm{~mm}$ electricity cable of 7 internal copper wires covered with a plastic shield. Peripheral region is unshielded and copper wires are adjusted to simulate hepatic arteries by expert radiologist as shown in Fig. 14(a). The metal wire was imaged 


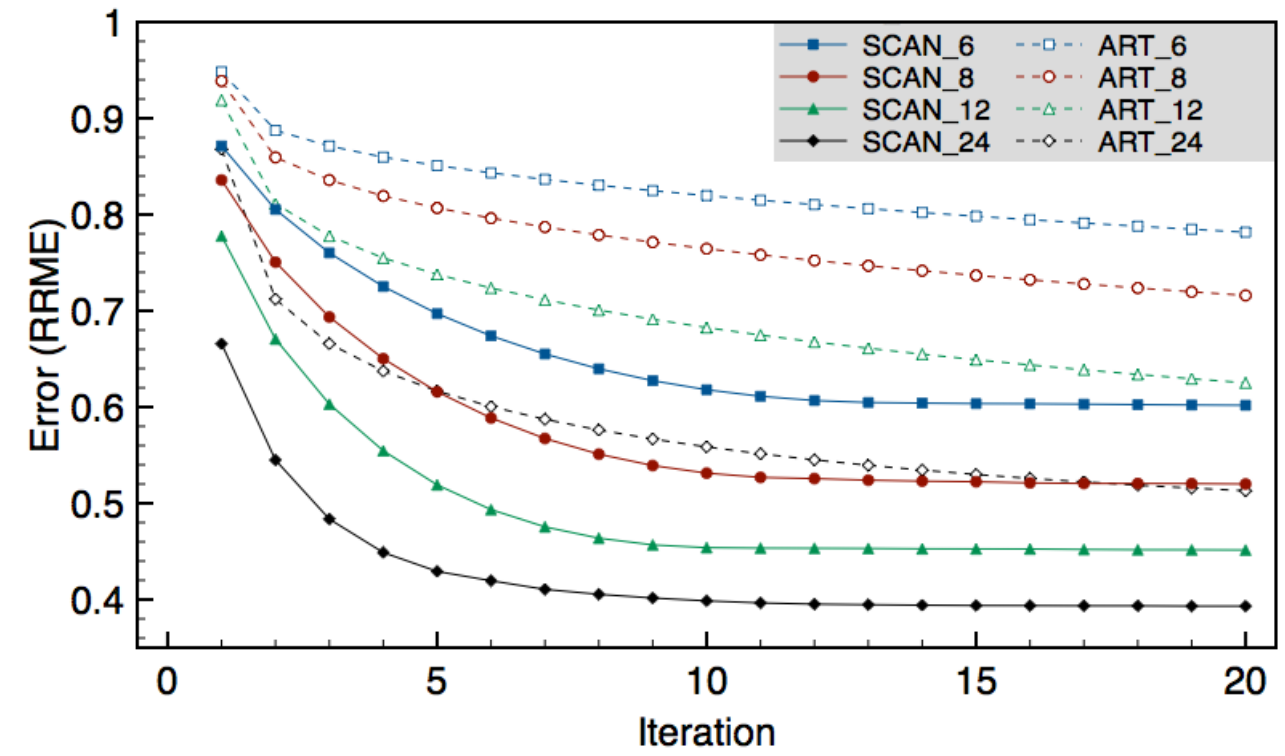

Figure 10: RRME value computed with different iterations using ART and SCAN algorithms for images reconstructed from different projection views. Results are associated with images shown in Fig. 9. 

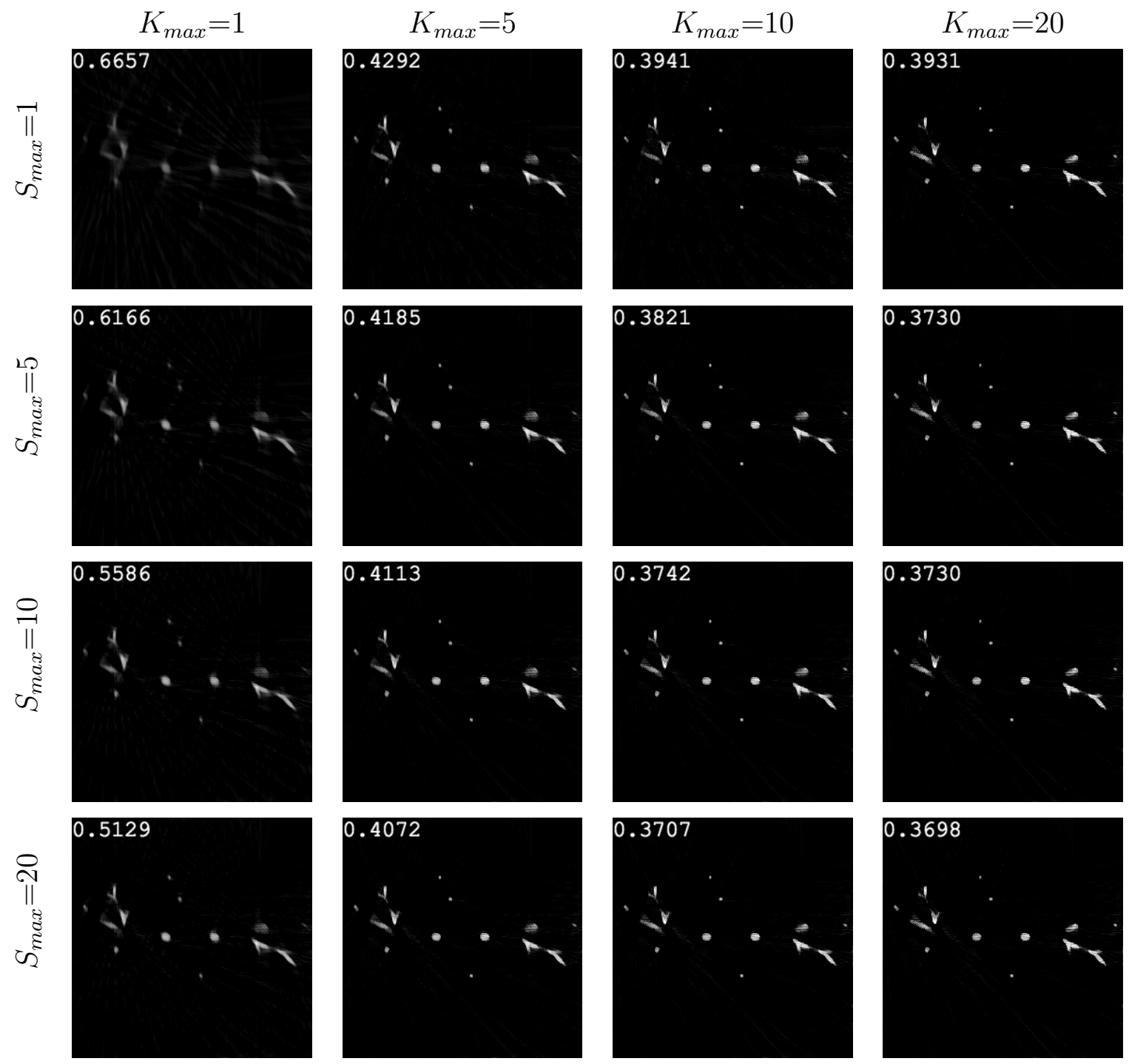

Figure 11: Central slices of SCAN reconstructions with fixed $\rho=20$ and different $K_{\max }$, $S_{\max }$ combinations. RRME value is shown in the top left corner of each image. 

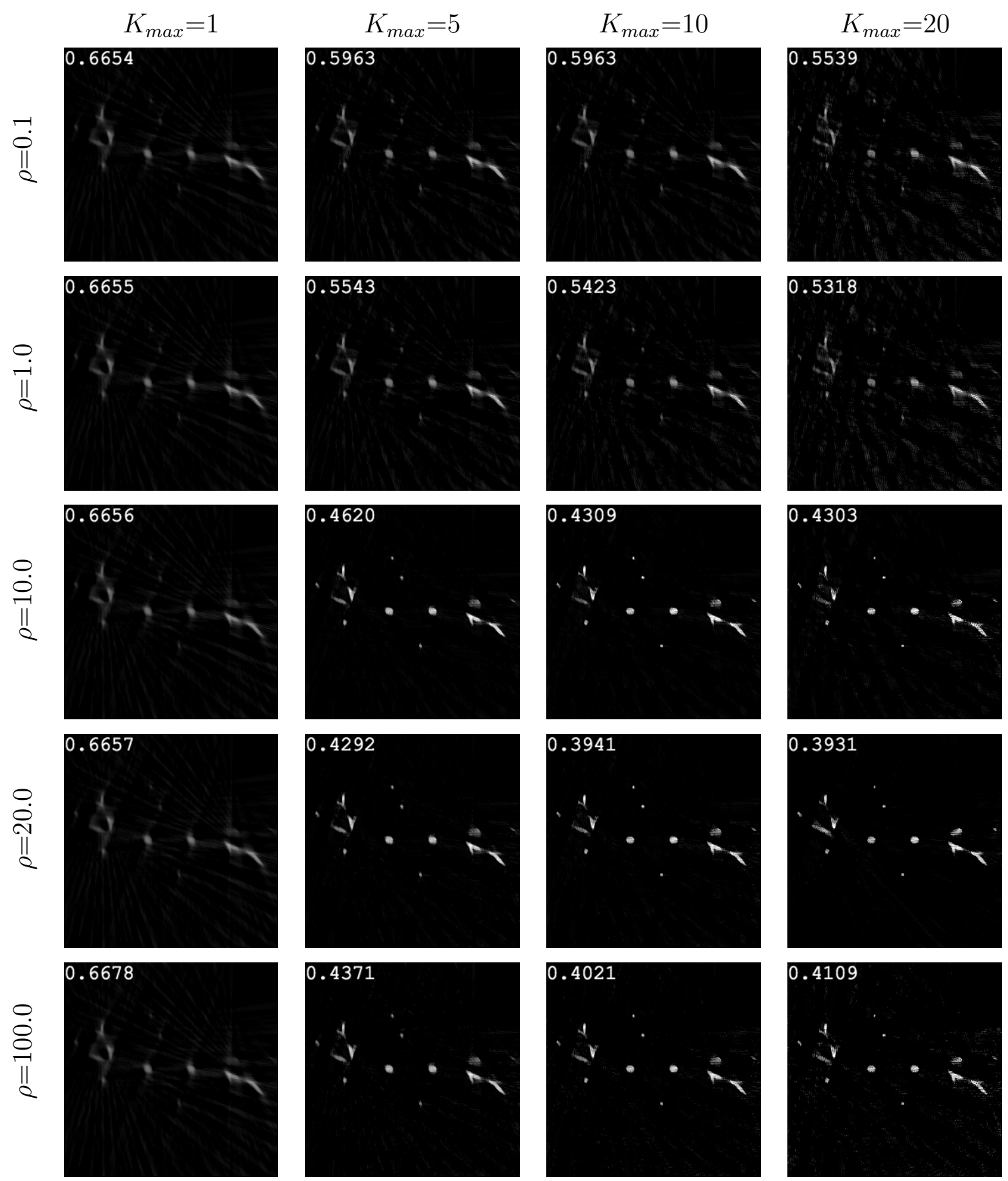

Figure 12: Central slices of SCAN reconstructions with fixed $S_{\max }=1$ and different $K_{\max }$, $\rho$ combinations. RRME value is shown in the top left corner of each image. 
(a) $\theta=0^{\circ}$

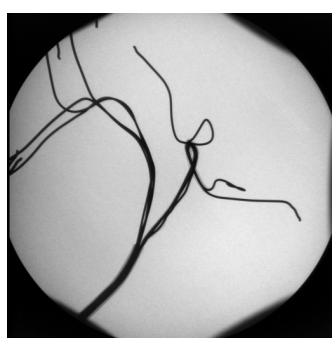

(e) $\theta=40^{\circ}$

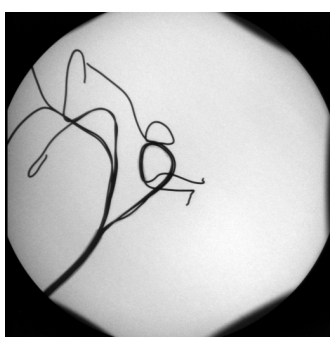

(i) $\theta=80^{\circ}$

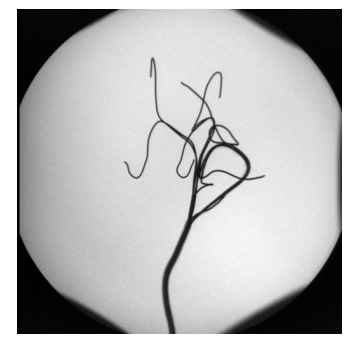

(j) $\theta=90^{\circ}$
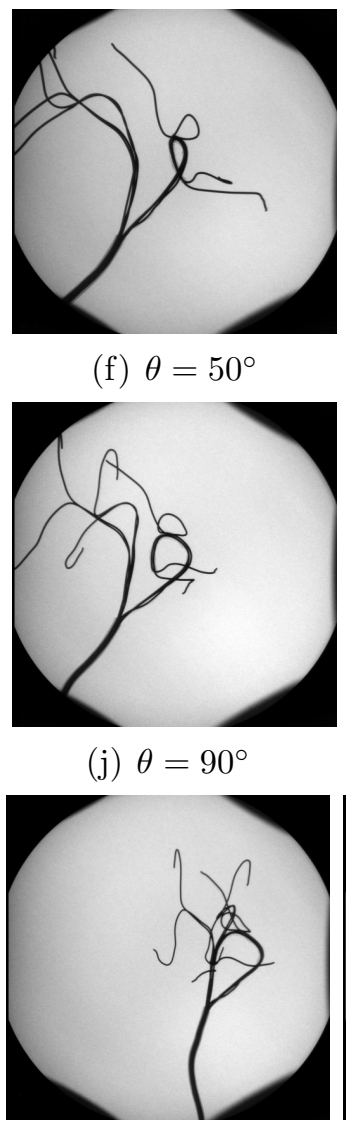

(f) $\theta=50^{\circ}$

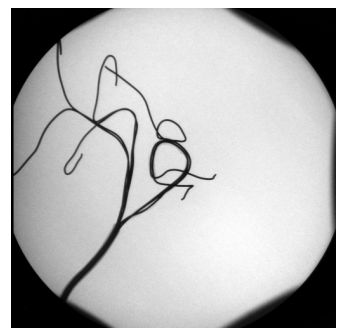

(g) $\theta=60^{\circ}$

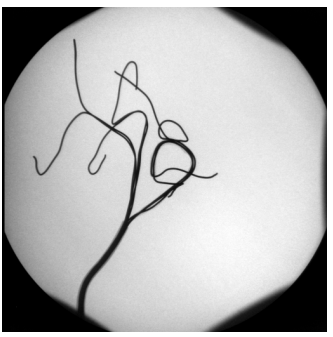

(k) $\theta=100^{\circ}$
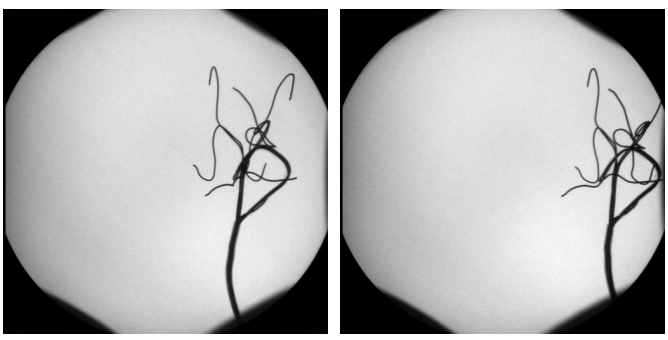

Figure 13: Real projection data acquired from metal wire phantom shown in Fig. 14(a) measured by the $\mathrm{C}$-arm detector in different view angles $\theta$. 
using C-arm scanner shown in Fig. 3(a). The scanner gantry was adjusted manually to acquire 24 projection views with angle spacing of $5^{\circ}$. Angle scale label attached to the scanner gantry is used to manually adjust gantry angulation with error around $\pm 0.1^{\circ}$. The wire phantom was located within approximate distance of $9.2 \mathrm{~cm}$ from the scanner center of rotation in both orthogonal Cartesian coordinates. This position was selected such that largest region of the object can be viewed in different scanner view angles as a result of the scanner geometry analysis shown in Fig. 4. Examples of acquired detector measurements, extracted in DICOM image format, are shown in Fig. 13. Projection data were calculated using blank scan measurements and image reconstruction was performed using the proposed algorithm with $\rho=10$,

$S_{\max }=1$ and $K_{\max }=25$. Volume rendering of the reconstructed image together with a photograph of the wire object are shown in Fig. 14. A video represents a $360^{\circ}$ rotation of the rendered 3D image is submitted as a supplementary material associated to this paper. Weak artifacts are observed in the image reconstructed from the real data. However, it is still in the acceptable quality for minimal invasive surgery procedures, where the most important task in these applications is to identify the 3D structure of the blood-vessels that is enough for catheter road-mapping in TACE procedure.

\section{Conclusion}

We proposed an iterative image reconstruction algorithm for 3D vascular imaging using a C-arm scanner. The developed algorithm was designed to overcome data limitation problems in this imaging modality including limited views, short orbit and offset scan geometries. This framework aims to 
(a) Real object

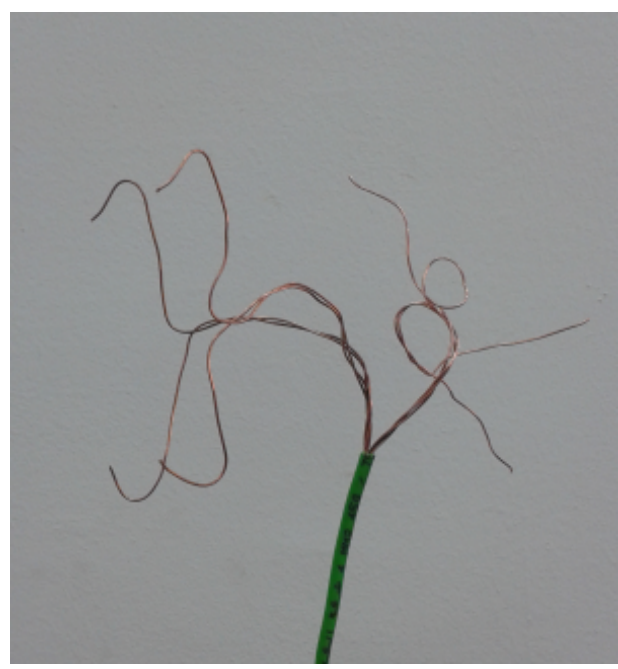

(b) Reconstructed 3D image

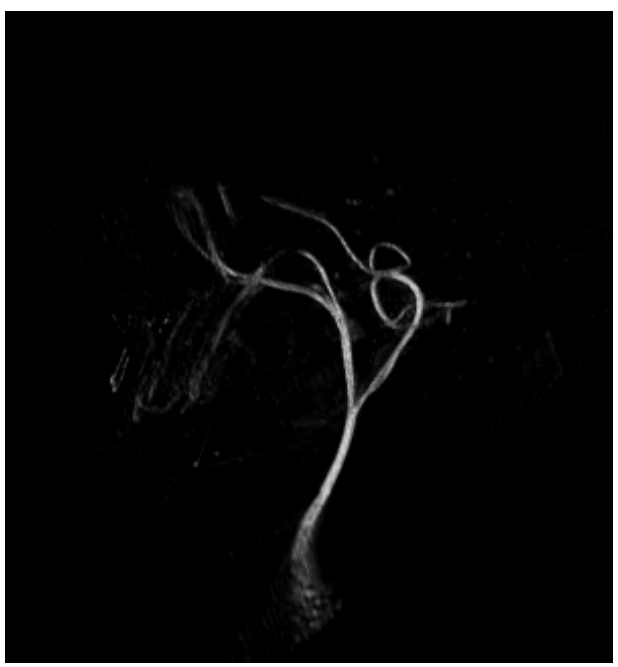

Figure 14: (a) Metal wire object used to simulate human segmental hepatic arterial system enhanced with contrast agent. (b) Three-dimensional volume rendering of reconstructed image of the metal wire from 24 projection views.

provide an effective 3D imaging using conventional C-arm scanners which are originally designed to generate $2 \mathrm{D}$ fluoroscopy images. The proposed imaging and reconstruction technique has several benefits: (1) it provides 3D images using conventional inexpensive equipments and (2) it improves the image quality obtained from highly down-sampled projection data, which is associated with patient dose level, interventional procedure time and amount of injected contrast agent. This work is an initial study and preliminary results using simulated and real data are encouraging. Future studies will include implementation of automatic data acquisition system and more accurate geometry calibration. In automatic data acquisition system, we are planning to automatically transfer the projection data from the scanner storage unit to the processing unit for $3 \mathrm{D}$ image reconstruction. In the current stage, the 
projection data are transferred manually. The target equipment was initially designed for $2 \mathrm{D}$ image acquisition rather than $3 \mathrm{D}$. It is important to conduct a comprehensive analysis of the gantry rotation error and perform system calibration for higher quality $3 \mathrm{D}$ reconstruction.

\section{Acknowledgements}

This work is partially supported by the Applied Scientific Research Projects (ASRP) program, Ministry of Higher Education, Egypt under Grant no. ASRP1-008-SUE.

\section{References}

[1] N. Strobel, O. Meissner, J. Boese, T. Brunner, B. Heigl, M. Hoheisel, G. Lauritsch, M. Nagel, M. Pfister, E.-P. Rührnschopf, et al., 3D imaging with flat-detector C-arm systems, in: Multislice CT, Springer, 2009, pp. $33-51$.

[2] J. A. Kaufman, M. J. Lee, Vascular and Interventional Radiology, Elsevier Health Sciences, 2013.

[3] P. S. Cho, A. D. Rudd, R. H. Johnson, Cone-beam CT from widthtruncated projections, Comput. Med. Imaging and Graph. 20 (1996) $49-57$.

[4] M. Li, H. Yang, H. Kudo, An accurate iterative reconstruction algorithm for sparse objects: application to 3D blood vessel reconstruction from a limited number of projections, Phys. Med. Biol. 47 (2002) 2599-2609. 
[5] V. Liu, N. R. Lariviere, G. Wang, X-ray micro-CT with a displaced detector array: Application to helical cone-beam reconstruction, Med. Phys. 30 (2003) 2758-2761.

[6] J. Siewerdsen, D. Moseley, S. Burch, S. Bisland, A. Bogaards, B. Wilson, D. Jaffray, Volume CT with a flat-panel detector on a mobile, isocentric C-arm: pre-clinical investigation in guidance of minimally invasive surgery, Med. Phys. 32 (2005) 241-254.

[7] G.-H. Chen, J. Zambelli, B. E. Nett, M. Supanich, C. Riddell, B. Belanger, C. A. Mistretta, Design and development of C-arm based conebeam CT for image-guided interventions: initial results, in: Proceedings of the SPIE, Medical Imaging 2006: Physics of Medical Imaging, vol. 6142, p. 614210.

[8] S. Sorensen, M. Mitschke, T. Solberg, Cone-beam CT using a mobile C-arm: a registration solution for IGRT with an optical tracking system, Phys. Med. Biol. 52 (2007) 3389-3404.

[9] D. Ritter, J. Orman, C. Schmidgunst, R. Graumann, 3D soft tissue imaging with a mobile C-arm, Comput. Med. Imaging and Graph. 31 (2007) $91-102$.

[10] E. A. Rashed, H. Kudo, Statistical image reconstruction from limited projection data with intensity priors, Phys. Med. Biol. 57 (2012) 20392061.

[11] X. Han, J. Bian, E. L. Ritman, E. Y. Sidky, X. Pan, Optimization-based 
reconstruction of sparse images from few-view projections, Phys. Med. Biol. 57 (2012) 5245-5273.

[12] D. L. Donoho, Compressed sensing, IEEE Trans Inf Theory 52 (2006) 1289-1306.

[13] E. J. Candès, J. Romberg, T. Tao, Robust uncertainty principles: Exact signal reconstruction from highly incomplete frequency information, IEEE Trans. Inf. Theory 52 (2006) 489-509.

[14] D. Gabay, B. Mercier, A dual algorithm for the solution of nonlinear variational problems via finite element approximation, Comput. Math. Appl. 2 (1976) 17-20.

[15] S. Ramani, J. Fessler, A splitting-based iterative algorithm for accelerated statistical X-ray CT reconstruction, IEEE Trans. Med. Imaging 31 (2012) 677-688.

[16] A. Sawatzky, Q. Xu, C. Schirra, M. Anastasio, Proximal ADMM for multi-channel image reconstruction in spectral X-ray CT, IEEE Transactions on Medical Imaging 33 (2014) 1657-1668.

[17] T. Hebert, R. Leahy, A generalized EM algorithm for 3-D Bayesian reconstruction from poisson data using Gibbs priors, IEEE Trans. Med. Imaging 8 (1989) 194-202.

[18] E. Y. Sidky, C.-M. Kao, X. Pan, Accurate image reconstruction from few-views and limited-angle data in divergent-beam CT, J. X-ray Sci. Technol. 14 (2006) 119-139. 
[19] S. Alenius, U. Ruotsalainen, Bayesian image reconstruction for emission tomography based on median root prior, Eur. J. Nucl. Med. 24 (1997) $258-265$.

[20] E. Hansis, D. Schafer, O. Dossel, M. Grass, Evaluation of iterative sparse object reconstruction from few projections for 3-D rotational coronary angiography, IEEE Trans. Med. Imaging 27 (2008) 1548-1555.

[21] E. Rashed, H. Kudo, Row-action image reconstruction algorithm using $\ell_{p}$-norm distance to a reference image, in: Nuclear Science Symposium and Medical Imaging Conference (NSS/MIC), 2011, IEEE, pp. 39663970.

[22] R. G. Baraniuk, Compressive sensing, IEEE Signal Process. Mag. 24 (2007).

[23] M. Fornasier, H. Rauhut, Compressive sensing, in: Handbook of Mathematical Methods in Imaging, Springer, 2011, pp. 187-228.

[24] E. A. Rashed, H. Kudo, Overview of compressed sensing approach to CT image reconstruction, Med. Imaging Technol. 31 (2013) 9-14.

[25] S. Leng, J. Tang, J. Zambelli, B. Nett, R. Tolakanahalli, G.-H. Chen, High temporal resolution and streak-free four-dimensional cone-beam computed tomography, Phys. Med. Biol. 53 (2008) 5653-5673.

[26] R. Tibshirani, Regression shrinkage and selection via the Lasso, J. R. Stat. Soc. Ser. B (Methodol.) (1996) 267-288. 
[27] A. C. Kak, M. Slaney, Algebraic reconstruction algorithms, in: Principles of Computerized Tomographic Imaging, SIAM, 2001, pp. 275-296.

[28] G. T. Herman, L. B. Meyer, Algebraic reconstruction techniques can be made computationally efficient, IEEE Trans. Med. Imaging 12 (1993) 600-609.

[29] C. Byrne, Accelerating the EMML algorithm and related iterative algorithms by rescaled block-iterative methods, IEEE Trans. Image Process. 7 (1998) 100-109.

[30] K. Mueller, R. Yagel, J. Wheller, Fast implementations of algebraic methods for three-dimensional reconstruction from cone-beam data, IEEE Trans. Med. Imaging 18 (1999) 538-548.

[31] H. Guan, R. Gordon, A projection access order for speedy convergence of ART (algebraic reconstruction technique): a multilevel scheme for computed tomography, Phys. Med. Biol. 39 (1994) 2005.

[32] S. Boyd, N. Parikh, E. Chu, B. Peleato, J. Eckstein, Distributed optimization and statistical learning via the alternating direction method of multipliers, Found. Trends Mach. Learn. 3 (2011) 1-122.

[33] R. T. Rockafellar, Convex analysis, Princeton University Press, 1997.

[34] D. L. Donoho, De-noising by soft-thresholding, IEEE Trans. Inf. Theory 41 (1995) 613-627.

[35] L. Elsner, I. Koltracht, P. Lancaster, Convergence properties of ART and SOR algorithms, Numer. Math. 59 (1991) 91-106. 
[36] G. T. Herman, Fundamentals of Computerized Tomography: Image Reconstruction from Projections, Springer, 2009. 